\title{
Investigando en los Tiempos de COVID-19: Re- inventando un Proyecto Participativo sobre el Autocuidado de la Diabetes en Adultos Mayores de Ontario, Canadá
}

\author{
Pilar Camargo-Plazas ${ }^{1}$, Idevania G. Costa ${ }^{2}$, Genevieve Pare', Beatriz \\ Alvarado $^{3}$ y Lenora Duhn ${ }^{1}$
}

\author{
${ }^{1}$ School of Nursing, Queen's University, Ontario, Canada | mdpc@queensu.ca \\ genevieve.pare@queensu.ca; duhnl@queensu.ca | https://orcid.org/0000-0002-8349-7723; \\ https://orcid.org/0000-0002-3714-9744; http://orcid.org/0000-0002-0401-953X \\ ${ }^{2}$ School of Nursing, Lakehead University, Ontario, Canada | idevania.costa@lakeheadu.ca \\ https://orcid.org/0000-0001-8024-2074 \\ ${ }^{3}$ Department of Public Health Sciences, Queen's University, Ontario, Canada \\ alvaradb@queensu.ca | https://orcid.org/0000-0002-1269-405X
}

\begin{abstract}
Resumen: EI COVID-19 ha afectado como vivimos, aprendemos, trabajamos e investigamos. Objetivos: Reflexionar en los efectos de la pandemia en nuestro trabajo de investigación participativa con adultos mayores que viven con diabetes. Métodos: Este estudio sigue un marco de investigación acción participativa (IAP) y una metodología de pedagogía crítica. Resultados: En este artículo reportamos nuestra experiencia como investigadores en un proyecto participativo durante la pandemia. Reflexionamos en los cambios que hemos implementado con el fin de continuar el proyecto a pesar de las restricciones. Conclusiones: Concluimos con lecciones aprendidas y una nueva visión sobre cómo mantener la naturaleza colaborativa de IAP en instancias en las que es imposible trabajar en estrecha proximidad con los miembros de la comunidad y realizar investigaciones en un determinado contexto.
\end{abstract}

Palabras clave: Investigación Acción Participativa; Pedagogía Crítica; COVID-19; Adultos mayores; Diabetes.

Conducting Research in the Time of COVID-19: Re-visioning a PAR Project about Diabetes Self-management Education for Seniors in Rural Ontario, Canada

\begin{abstract}
The impact of the global COVID-19 pandemic cannot be overstated, affecting how we live, learn, work, and do research. Goals: Reflect on the impact of the global pandemic in a participatory research project with seniors living with diabetes. Method: This study follows a participatory action research and critical pedagogy approaches. Results: In this paper, we describe our experience as researchers working on a PAR project during the pandemic. We reflect on the challenges, the research modifications made in our efforts to move forward with distance-based participatory approaches, and the surprising (unimagined) benefits Conclusions: We conclude with lessons learned and new visioning about how to maintain the collaborative nature of PAR in instances where it is impossible to work in close proximity with community members and conduct research in a co-located context.
\end{abstract}

Keywords: Participatory Action Research; Critical Pedagogy: COVID-19; Seniors; Diabetes.

\section{Introducción}

Hasta mayo 10 del 2021, cerca de 158 millones de personas fueron diagnosticadas con el COVID-19 y cerca de 3.29 millones fallecieron a causa de esta enfermedad (WHO, 2021). Durante la misma fecha, en Canadá, 1.29 millones de casos de COVID-19 se reportaron y 24.626 canadienses han fallecido a causa del COVID-19 (Government of Canada, 2021). Aunque la pandemia ha afectado la vida de muchos canadienses, los adultos mayores han sido identificados como una población particularmente vulnerable. Según el gobierno del Canadá (2021), un $20 \%$ de los adultos mayores canadienses se han visto afectados por COVID-19. Además, el $36 \%$ de las muertes se han producido entre las personas de 65 a 84 años y en un $52 \%$ en los adultos mayores de 85 años (Statistics Canada, 2021). La pandemia de COVID-19 ha afectado de forma particular a los adultos mayores con enfermedades crónicas como la diabetes (Bornstein et al., 2020; Guo et al., 2020; Javanmardi et al., 2020). 
Las personas que viven con diabetes tienen más probabilidades de sufrir complicaciones como la neumonía o morir durante la hospitalización a causa del COVID-19. Esta información es particularmente relevante en la provincia de Ontario, en donde las estadísticas indican que la diabetes se ha incrementado en la población de adultos mayores. Por ejemplo, en el 2018, 437.800 personas de 65 años fueron diagnosticadas con diabetes, cifra que aumentó a 513.500 personas en el 2019 (Statistics Canada, 2021). Por consiguiente, la pandemia ha afectado a las comunidades vulnerables como las personas mayores que viven con diabetes, siendo este grupo, uno de los grupos a los cuales va dirigido el desarrollo de proyectos de investigación participativa (Valdez \& Gubrium, 2020).

Durante el último año hemos vivido variantes emergentes del virus, la amenaza constante de brotes en grupos vulnerables como los adultos mayores con diabetes y la complejidad de la distribución de la vacuna (Santana et al., 2021). Sin embargo, lo más importante es que la pandemia ha dejado al descubierto inequidades sociales y de salud experimentados por grupos poblacionales vulnerables (Valdez \& Gubrium, 2020). La investigación de acción participativa (IAP), entonces, toma un rol especialmente importante durante la pandemia al explorar problemas de injusticia social revelados en el último año. Una de las características importantes de IAP es la interacción personal y constante entre miembros de la academia, miembros de la comunidad y organizaciones en el proceso de planeación, diseño, implementación, evaluación, movilización de conocimiento y sostenibilidad de iniciativas de investigación (Valdez \& Gubrium, 2020). Tales prácticas son imposibles durante la pandemia debido a las restricciones impuestas para evitar la propagación del COVID-19 y sus variantes.

Entonces, bajo medidas de distanciamiento físico y con deficiencia en el acceso a la tecnología ¿cómo podemos asegurar la equidad, la apertura y la participación en un proyecto IAP durante COVID-19? ¿Cómo puede un investigador involucrar a los miembros de la comunidad durante la pandemia, especialmente durante estos momentos tan estresantes? ¿Cómo se pueden mantener relaciones de igualdad de poder entre investigadores y participantes si ambos estamos dispersos en diferentes ubicaciones geográficas o no tenemos acceso a Internet confiable? En este artículo presentamos nuestras respuestas a cada una de estas preguntas, usando como ejemplo un estudio de IAP con adultos mayores que viven con diabetes en Sharbot Lake, Ontario. A continuación, presentamos una descripción del estudio, seguimos con información sobre la metodología del proyecto, reflexión sobre el proceso de investigación durante la pandemia y concluimos con lo que hemos aprendido a través de este proceso.

\section{Descripción del Estudio}

Al corazón de este trabajo ha estado el considerar las voces de las personas mayores en el desarrollo de programas de educación enfocados en el autocuidado de la diabetes. En la literatura explorada, no hemos encontrado estudios que involucren a los adultos mayores y proveedores como colaboradores en el proceso de planeación, diseño, implementación y evaluación de programas de educación en el autocuidado de la diabetes(Camargo-Plazas, et al., 2020). Nuestro trabajo está orientado a abordar esta brecha de conocimiento. Siguiendo los lineamientos y filosofías de la investigación acción participativa y de la pedagogía crítica, planeamos invitar a un grupo de adultos mayores a hablar sobre sus experiencias de autocuidado de la diabetes. Inicialmente habíamos propuesto una consulta con la comunidad, en donde a través de un ejercicio de foto voz ellos identificarían sus necesidades de educación para el autocuidado de la diabetes. Originalmente, 30 adultos mayores iban a ser invitadas en abril del 2020 a la consulta. Sin embargo, debido a COVID-19 nuestro trabajo quedo suspendido. En la actualidad y por razones que explicamos detalladamente en otras secciones de este artículo, hemos decidido invitar a solo 10 adultos mayores de 65 años de la comunidad a participar en una actividad de foto voz y una entrevista telefónica. Hasta el día de hoy hemos reclutado ocho participantes. Este estudio ha sido aprobado por el comité de ética de Queen's University. Para el análisis de datos, hemos pensado seguir el marco teórico de Loppie-Reading and Wien (2013), sobre los determinantes sociales de la salud. 
Ellos clasifican los determinantes como proximales (ej. Hábitos de vida, estatus socioeconómico, medio ambiente, etc.), intermedios (ej. Sistema de salud, infraestructuras y recursos en las comunidades) y distales (históricos, políticos, gobierno, etc.), cada uno de ellos con unos factores que ayudan a crear inequidades de salud en grupos poblacionales vulnerables (Camargo-Plazas et al., 2020). Una vez las medidas de restricción se levanten, queremos invitar a esos participantes a ser parte de un círculo de cultura para conversar sobre los temas encontrados en las entrevistas individuales. Más adelante en las siguientes secciones reflexionamos acerca de nuestras experiencias con este proyecto y como cada actividad ha sido replanteada para acomodar el estudio a la realidad de la pandemia.

\subsection{Objetivo del Estudio}

El objetivo de este estudio es examinar como los determinantes sociales de la salud afectan la educación para el autocuidado de la diabetes en los adultos mayores. Otro objetivo es el de invitar a las personas mayores, a los profesionales de salud y miembros de la comunidad interesados a la creación de soluciones colectivas y sostenibles que consideren a través de enfoques alternativos las necesidades de educación en salud de esta población.

\subsection{Organización Comunitaria}

En colaboración con Sharbot Lake Family Health Team (SLFHT) estamos desarrollando este estudio. SLFHT es un proveedor de servicios pequeño ubicado en el pueblo de Sharbot Lake que pertenece al municipio de Central Frontenac, en el sureste de Ontario, Canadá. Igualmente, SLFHT también brinda servicios a los residentes de las regiones de North and South Frontenac con un total aproximado de 2.700 usuarios registrados. Según los datos del censo más actual, la población de Central Frontenac es de aproximadamente 4.370 habitantes, con 1.155 personas de 65 años o más (Statistics Canada, 2017). La prevalencia de ingresos bajos en el municipio es del 19\% con una taza para los hombres del $18.5 \%$ y de $19.5 \%$ para las mujeres. Según se reportó en el 2015 , casi el $17 \%$ de las personas de 25 a 64 años informa no tener educación, certificado, diploma o título (Statistics Canada, 2017). En el 2016, la prevalencia de la diabetes aumentó en la región sureste de Ontario con un 22.7\% para las personas entre los $60-69$ años y un $31.7 \%$ para los mayores de 80 años. Además, el número de personas con diabetes en los mismos grupos de edad se duplicó entre el 2006 y el 2016 aumentando un $90 \%$ cada uno (A. Mask, Ministry of Health And Long-Term Care Health Analytic Branch, personal communication, August 27, 2018). Aunque SLFHT tiene un programa de diabetes, la directora y el personal de salud están interesados en escuchar a los adultos mayores de la comunidad y particularmente sus propuestas para la educación en el autocuidado de la diabetes. El apoyo incondicional de la directora ejecutiva y de todo el personal de salud de SLFHT ha sido fundamental para el avance de este proyecto.

\subsection{Comité Asesor}

Dado nuestro enfoque de investigación participativa, el establecimiento de un comité asesor ha sido fundamental (Camargo-Plazas et al., 2020). Desde su inició decidimos que nuestra filosofía de trabajo seguiría la guía de un comité asesor. El comité asesor está constituido por un equipo de voluntarios, quienes trabajan en el proyecto garantizando que las perspectivas de los miembros de la comunidad de Sharbot Lake estén presentes en el proceso de investigación (Camargo-Plazas et al., 2020). El comité asesor incluye: 1) una administradora general de SLFHT; 2) un representante de la comunidad que vive con diabetes; 3) una enfermera y educadora certificada en diabetes de SLFHT; 4) la directora de planeación e integración de la región sureste de Ontario; 5) una NP especializada en el cuidado de la diabetes; y 6) una dietista registrada y educadora certificada en diabetes de SLFHT. 
Nuestra primera reunión tuvo lugar en julio de 2019, desde entonces hemos tenido reuniones casi cada mes para presentar avances o buscar la opinión sobre dificultades encontradas con el proyecto. En el futuro, las reuniones continuarán para asegurarnos de mantener a los miembros regularmente informados y brindar la oportunidad de conversar sobre temas que quizás no hayamos considerado o no consideremos necesarios.

\section{Metodología}

Nuestro estudio sigue un enfoque de investigación acción participativa (IAP) (MacKinnon, 2018) y una metodología de pedagogía crítica (Freire, 2013). IAP está alineada a filosofías de participación, colaboración y empoderamiento que involucran a miembros de la comunidad y organizaciones comunitarias en la coproducción de conocimiento para el cambio social (Chapman, 2019; Kemmis et al., 2014; MacKinnon, 2018). En IAP, la colaboración de miembros de la comunidad comienza desde el planteamiento del problema hasta la movilización de resultados a través de diálogo y un proceso iterativo de investigación (Chapman, 2019). IAP sigue un ciclo iterativo dinámico, recursivo de acción, investigación y reflexión (Chapman, 2019; MacKinnon, 2018). A través de la participación equitativa, la participación democrática y la libertad, se busca en IAP alcanzar el potencial humano (Kemmis et al., 2014). Un marco IAP guía nuestro estudio y proporciona un punto de partida para construir colaboraciones efectivas con miembros de la comunidad y organizaciones interesadas en la coproducción de intervenciones de educación para el autocuidado de la diabetes en personas mayores de Ontario, Canadá.

Desarrollado por el educador brasileño Paulo Freire $(2002,2013)$, la pedagogía crítica se preocupa por crear un proceso de concientización de los estudiantes para transformar sus vidas y condiciones a través del aprendizaje (Freire, 2002, 2013). En la pedagogía crítica, los seres humanos rompen con el proceso educativo tradicional en donde el educador deposita conocimiento en los estudiantes (Freire, 2002). Por el contrario, en la pedagogía crítica, la educación debe generar un ser humano nuevo, consciente de su realidad y comprometido con su transformación y cambio (Freire, 2002, 2013). El tener un enfoque de pedagogía crítica en nuestro estudio nos ayudara a abordar la educación para el autocuidado de la diabetes de una forma diferente, en donde los adultos mayores serán participantes activos en su proceso de aprendizaje. Igualmente, la pedagogía crítica nos permitirá comprender las vivencias de las personas mayores que viven con diabetes y los efectos que tienen los determinantes sociales de la salud en su proceso de autocuidado. Entonces, es a través de un proceso de concientización que se planificarán las acciones necesarias para mejorar la calidad de vida de esta población.

\section{Nuestra Experiencia de Investigación durante COVID-19}

En marzo de 2020 el mundo cambió para todos. COVID-19 ha afectado sin precedentes la forma en que vivimos, trabajamos e investigamos (Hall, 2020). Hoy en día, nuestra rutina diaria incluye el distanciamiento físico de otras personas que no viven con nosotros, evitar multitudes, usar una máscara en espacios públicos o cerrados, cuarentenas, lavarnos las manos con frecuencia, trabajar desde casa y limitar los viajes innecesarios. Todos estos cambios ocurrieron en el espacio de unas pocas semanas y afectan nuestras capacidades personales, sociales y profesionales. Las nuevas restricciones han impactado dramáticamente los proyectos de investigación que siguen un enfoque IAP, en donde el objetivo es trabajar en estrecha colaboración con miembros de una comunidad para coproducir conocimiento y cambio social (Kemmis et al., 2014; MacKinnon, 2018). Investigadores que siguen un enfoque participativo han usado métodos como las consultas comunitarias, el World Café, teatro popular, grupos focales, foto voz y círculos culturales para crear y afianzar vínculos con miembros de una comunidad determinada (CamargoPlazas, Costa, Alvarado, et al., 2020; Cameron et al., 2014). Además de restringir las interacciones en persona con los posibles participantes del estudio, la pandemia ha limitado nuestras interacciones con otros investigadores, organizaciones comunitarias y otras partes interesadas. 
Nuestro proyecto, titulado: "Revisión de la educación para el autocuidado de las personas mayores que viven con diabetes" se planeó inicialmente como una consulta comunitaria con miembros de la comunidad, personas interesadas como (ej. médicos, educadores, tomadores de decisiones, administradores de atención médica y líderes comunitarios), e investigadores, para determinar las formas más efectivas de abordar la educación para el autocuidado de las personas mayores con diabetes.

Antes del inicio de la pandemia y bajo la dirección de nuestro comité asesor, refinamos nuestras actividades de investigación para utilizar la foto voz y los círculos culturales como nuestros métodos de participación. Con el método de foto voz, nuestro objetivo es poner cámaras en manos de las personas mayores que viven con diabetes, para documentar su vida diaria y sus experiencias de autocuidado (Evans-Agnew \& Rosemberg, 2016). Después de la foto voz, planeábamos invitar a las personas mayores a participar en círculos culturales (Freire, 2013) como un método de concientización para explorar los problemas representados por las fotos de los participantes. Sin embargo, estos métodos de participación tuvieron que ser modificados o reemplazados por completo durante la pandemia, ya que dependían de una interacción cara a cara con personas a riesgo de adquirir COVID-19, como lo son los adultos mayores que viven con diabetes. En diálogo con nuestro comité asesor, creamos formas de avanzar el proyecto durante la pandemia. Se decidió como grupo el retomar la foto voz y el implementar entrevistas individuales y omitir los círculos de cultura hasta cuando se levanten las restricciones. Originalmente, planeábamos invitar a 30 adultos mayores a participar en el estudio, dadas las restricciones de movilización el comité asesor decidió reducir el número de participantes a solo 15 .

Cada jueves una parte del equipo de investigación que constaba de las dos investigadoras principales y la coordinadora del proyecto, nos reuníamos por una hora a planear la logística de las actividades de investigación. No obstante, nuevos obstáculos comenzaron a emerger, por ejemplo, nos preguntábamos que tipo de cámaras debíamos utilizar, si serían mejor las cámaras desechables o comprar unas cámaras digitales que sirvieran en otros proyectos donde empleamos foto voz. Cada jueves al igual que nuevos desafíos emergían, se encontraba como grupo respuestas. El problema de las cámaras fue resuelto cuando la coordinadora del proyecto nos comentó acerca de unas iPad del laboratorio de la escuela de enfermería donde trabajamos que habían sido dadas de baja por viejas. Ella las rescató y las dejó para uso en investigación. En cuánto a como conectarnos con los participantes, se planteó inicialmente el uso de Zoom como una plataforma confiable para conectarnos con nuestros participantes en la investigación. Planeamos cada detalle, y sin embargo, nuevos retos emergían en cada reunión. Por ejemplo, no sabíamos si el acceso a internet en el área rural donde nuestra investigación tendría lugar era adecuado y disponible en la comunidad. También, comenzamos a cuestionar la alfabetización tecnológica de la comunidad. En un momento determinado invitamos a la representante de la comunidad en nuestro comité asesor quien es la directora del programa de alfabetización para mayores en la comunidad donde trabajamos. Ella asistió a una de nuestras reuniones y nos respondió a cada uno de nuestros interrogantes. Igualmente, nos confirmó nuestras sospechas acerca de los desafíos y las barreras en esa comunidad, las cuales incluían la falta de alfabetización tecnológica en las personas mayores, la confiabilidad de internet en el entorno rural y las preocupaciones financieras que limitan el acceso al internet de alta velocidad e ilimitado. Ella, igual, planteó la idea de realizar entrevistas telefónicas en vez de usar Zoom para no excluir a miembros de la comunidad interesados en el proyecto, pero sin acceso o con acceso limitado al internet.

Era igualmente importante para nosotras el contar con la perspectiva de esta líder de la comunidad quien muy amablemente contesto cada una de nuestras preguntas. Es más, ella nos ayudó a mejorar el nivel de lenguaje de todos los documentos a los que van a tener acceso los participantes del proyecto. Como miembro de la comunidad y a través de su centro de alfabetización, ella ofrecerá respuesta a los participantes sobre temas relacionados con la toma de fotos o el manejo del iPad. En este plan revisado, el reclutamiento inicial lo realiza una enfermera profesional y miembro de nuestro comité asesor, quien se comunica con los posibles participantes en su práctica de control del programa de diabetes. 
Una vez ella identifica a los potenciales participantes, un miembro del equipo se conecta con ellos por teléfono para explicar el proyecto en profundidad y obtener consentimiento verbal. Cuando la persona decide que quiere participar, se le envía por correo certificado el material de estudio el cual incluye: los formularios de consentimiento, una guía para la toma de fotos, un iPad con un video de bienvenida, cargador y cable, así como instrucciones sobre el uso del iPad, el teléfono y correo de nuestra líder comunitaria por si tienen preguntas sobre el iPad y un sobre prepagado para devolución del iPad. Terminada la toma de fotos los participantes devolverán el iPad y la coordinadora del proyecto imprimirá las fotos digitales y enviará las fotos de regreso a los participantes con un identificador en la parte de atrás para poder facilitar la entrevista por teléfono.

Además de lo expresado arriba, esta experiencia nos ha enseñado lo importante que es la ubicación geográfica, alfabetización tecnológica y el acceso a la tecnología como determinantes sociales de la salud. El acceso en este caso a la comunicación en tiempo de una pandemia. Durante la pandemia muchos hogares han usado el internet para trabajar, comprar comida, telemedicina y comunicarse con la familia. Entonces el no tener un acceso a internet adecuado se presenta como un desafío significativo para los miembros de la comunidad. En alguna de nuestras discusiones incluso planteamos la posibilidad de comprar internet móvil, pero ninguna de las compañías que consultamos ofrecía servicio en el municipio de Sharbot Lake. Otro aspecto importante para considerar en nuestro estudio es la experiencia del autocuidado de la diabetes en el adulto mayor durante COVID-19. El uso de la telemedicina durante la pandemia y la alfabetización en salud son barreras que deben ser exploradas de forma profunda en nuestro proyecto. Igualmente, esta experiencia nos ha hecho replantear nuestro estudio en varias oportunidades, lo que inicialmente se planteó como un proyecto de diálogo participativo se modificó. Sin embargo, al centro de nuestro estudio siempre han estado las filosofías de participación, colaboración y concientización que esperamos generar a través del estudio. Finalmente guardamos la esperanza de implementar los círculos de cultura en donde las personas mayores identifiquen los problemas y creen soluciones necesarias para mejorar su calidad de vida.

\section{Aprendizaje y Reflexión Final}

Este estudio de investigación se formuló como una iniciativa de base para abordar las necesidades de educación para el autocuidado de la diabetes en personas mayores. El camino ha sido largo y arduo. Sin duda, como muchos equipos de investigación hemos enfrentado desafíos y retrasos en el lanzamiento del proyecto debido a la pandemia. Sin embargo, hemos aprendido a ser flexibles y acomodar nuestro trabajo a la realidad de la pandemia. También hemos aprendido a salir de nuestro mundo de la investigación y mirar la realidad de las comunidades donde trabajamos. El desafío más importante de este trabajo de IAP ha sido el adaptar el proyecto a los efectos que ejercen los determinantes sociales de la salud en el proceso de investigación. La ubicación geográfica de la comunidad, el acceso a tecnología, el nivel de alfabetización tecnológica de las personas mayores son factores que inicialmente no consideramos, pero que realmente han afectado el desarrollo de este proyecto. Estas inequidades sociales crean un llamamiento ético y político para tomar medidas y cerrar esta brecha. Confiamos en que nuestro estudio, sea un primer paso para lograr ese fin. Este trabajo no habría podido suceder sin el apoyo incondicional y el trabajo arduo de nuestro comité asesor y los miembros de la comunidad. Esta es nuestra mayor recompensa, el trabajar de forma cercana con miembros líderes de la comunidad. A través de este año nos hemos apoyado en estas personas para crear un proyecto adaptado a las circunstancias de vida de las personas mayores del municipio de Sharbot Lake. Ellos han dedicado tiempo para orientarnos en como abordar este proyecto de una forma inclusiva. Finalmente, el realizar una investigación participativa durante COVID-19 implica adaptarse a los cambios inesperados del día a día, sin perder la visión y la filosofía de participación, colaboración y concientización que esperamos generar a través de nuestro estudio. 


\section{Agradecimientos}

Expresamos nuestros muy sinceros agradecimientos a la Dra. Deborah Tregunno por su inmensa colaboración en este proyecto. Igualmente agradecemos al personal de salud y administrativo de Sharbot Lake Family Health Team por su tiempo y dedicación en este estudio sin ustedes nada de esto sería posible. También agradecemos a los miembros de nuestro comité asesor quienes nos han dado ánimo y esperanza cuando el camino parecía difícil de continuar. Finalmente agradecemos a Canadian Institute of Health ResearchInstitute of Aging por el financiamiento de este proyecto.

\section{Referencias}

Bornstein, S. R., Rubino, F., Khunti, K., Mingrone, G., Hopkins, D., Birkenfeld, A. L., Boehm, B., Amiel, S., Holt, R. I., Skyler, J. S., DeVries, J. H., Renard, E., Eckel, R. H., Zimmet, P., Alberti, K. G., Vidal, J., Geloneze, B., Chan, J. C., Ji, L., \& Ludwig, B. (2020). Practical recommendations for the management of diabetes in patients with COVID-19. Lancet Diabetes Endocrinol, 8(6), 546-550. https://doi.org/10.1016/S2213-8587(20)30152-2

Camargo-Plazas, P., Costa, I., Duhn, L., Alvarado, B., \& Tregunno, D. (2020). Uso de métodos participativos en el desarrollo de un programa de educación para el autocuidado de la diabetes en adultos mayores en Canadá. Qualitative Research in Health: Advances and challenges, 3, 833-845.

Camargo-Plazas, P., Costa, I. G., Alvarado, B., Duhn, L., \& Tregunno, D. (2020). Adapting Paulo Freire's participatory education to develop self-management programs for seniors with diabetes. Canadian Journal of Diabetes, 1-4. https://doi.org/DOI:https://doi.org/10.1016/j.jcjd.2020.10.017

Cameron, B. L., Carmargo Plazas Mdel, P., Salas, A. S., Bourque Bearskin, R. L., \& Hungler, K. (2014, Jul-Sep). Understanding inequalities in access to health care services for aboriginal people: a call for nursing action. ANS Adv Nurs Sci, 37(3), E1-E16. https://doi.org/10.1097/ANS.0000000000000039

Chapman, M. (2019). Changing the world without doing harm: Critical pedagogy, participatory action research and the insider student researcher. Religious Studies and Theology, 38(12), 100-116. https://doi.org/https://doi.org/10.1558/rsth.38715

Evans-Agnew, R. A., \& Rosemberg, M. A. S. (2016). Questioning photovoice research: Whose voice? . Qualitative Health Research, 26(8), 1019-1030. https://doi.org/https://doi.org/10.1177/1049732315624223

Freire, P. (2002). Pedagogy of the oppressed. Bloomsbury.

Freire, P. (2013). Education for critical consciousness. Bloomsbury Academic.

Government of Canada. (2021). Coronavirus disease 2019 (COVID-19): Epidemiology update. https://health-infobase.canada.ca/src/data/covidLive/Epidemiological-summary-of-COVID19-cases-in-Canada-Canada.ca.pdf

Guo, W., Li, M., Dong, Y., Zhou, H., Zhang, Z., Tian, C., Qin, R., Wang, H., Shen, Y., Du, K., Zhao, L., Fan, H., Luo, S., \& Hu, D. (2020). Diabetes is a risk factor for the progression and prognosis of COVID-19. Diabetes/Metabolism Research and Reviews, 36(7), e3319-n/a. . https://doi.org/https://doi.org/10.1002/dmrr.3319

Hall, J. (2020). Taking part from a distance: Doing participatory research during Covid-19, an IET literature review http://www.open.ac.uk/blogs/opentel/taking-part-from-a-distance-doingparticipatory-research-during-covid-19-an-iet-literature-review/

Javanmardi, F., Keshavarzi, A., Akbari, A., Emami, A., \& Pirbonyeh, N. (2020). Prevalence of underlying diseases in died cases of COVID-19: A systematic review and meta-analysis. PLoS One, 15(10), e0241265. https://doi.org/10.1371/journal.pone.0241265

Kemmis, S., McTaggart, R., \& Nixon, R. (2014). The action research planner doing critical participatory action research. Springer Singapore. https://doi.org/https://doi.org/10.1007/978981-4560-67-2 
Vol. 8 | Investigación Cualitativa en Salud: Avances y Desafíos

Loppie-Reading, C., \& Wien, F. (2013). Health inequalities and social determinants of Aboriginal people's health. National Collaborating Centre for Aboriginal Health (NCCAH). https://www.nccih.ca/docs/determinants/RPT-HealthInequalities-Reading-Wien-EN.pdf

MacKinnon, S. (2018). Practising community-based participatory research: Stories of engagement, empowerment, and mobilization. Purich Books.

Santana, F., Hammond Wagner, C., Berlin Rubin, N., Bloomfield, L., Bower, E., Fischer, S., Santos, B., Smith, G., Muraida, C., \& Wong-Parodi, G. (2021). A path forward for qualitative research on sustainability in the COVID-19 pandemic. Sustainability Science, 1-7. https://doi.org/https://doi.org/10.1007/s11625-020-00894-8

Statistics Canada. (2017). Central Frontenac, TP [census subdivision], Ontario and Ontario [Province] (table). Census Profile. 2016 Census. Statistics Canada Catalogue no. 98-316X2016001. https://www12.statcan.gc.ca/census-recensement/2016/dp$\mathrm{pd} /$ prof/index.cfm?Lang=E

Statistics Canada. (2021). Table 13-10-0096-01 Health characteristics, annual estimates. https://doi.org/10.25318/1310009601-eng

Valdez, E., \& Gubrium, A. (2020). Shifting to virtual CBPR protocols in the time of corona virus/COVID-19. International Journal of Qualitative Method, 19. https://doi.org/https://doi.org/10.1177/1609406920977315 